\title{
Research Article \\ Critical Point Theorems and Ekeland Type Variational Principle with Applications
}

\author{
Lai-Jiu Lin, ${ }^{1}$ Sung-Yu Wang, ${ }^{1}$ and Qamrul Hasan Ansari ${ }^{2}$ \\ ${ }^{1}$ Department of Mathematics, National Changhua University of Education, Changhua 50058, China \\ ${ }^{2}$ Department of Mathematics, Aligarh Muslim University, Aligarh 202 002, Taiwan
}

Correspondence should be addressed to Lai-Jiu Lin, maljlin@cc.ncue.edu.tw

Received 28 September 2010; Accepted 9 December 2010

Academic Editor: S. Al-Homidan

Copyright (c) 2011 Lai-Jiu Lin et al. This is an open access article distributed under the Creative Commons Attribution License, which permits unrestricted use, distribution, and reproduction in any medium, provided the original work is properly cited.

\begin{abstract}
We introduce the notion of $\lambda$-spaces which is much weaker than cone metric spaces defined by Huang and X. Zhang (2007). We establish some critical point theorems in the setting of $\lambda$-spaces and, in particular, in the setting of complete cone metric spaces. Our results generalize the critical point theorem proposed by Dancs et al. (1983) and the results given by Khanh and Quy (2010) to $\lambda$-spaces and cone metric spaces. As applications of our results, we characterize the completeness of $\lambda$-space (cone metric spaces and quasimetric spaces are special cases of $\lambda$-space) and studying the Ekeland type variational principle for single variable vector-valued functions as well as for multivalued bifunctions in the setting of cone metric spaces.
\end{abstract}

\section{Introduction}

In the last three decades, the famous Ekeland's variational principle (in short, EVP) [1] (see also, $[2,3])$ emerged as one of the most important tools and results in nonlinear analysis due to its wide applications in optimization, optimal control theory, game theory, nonlinear equations, dynamical systems, and so forth; see, for example, [2-8] and references therein. It has been extended and generalized in different directions and in different settings. See, for example, [4, 5, 7-22] and references therein. The vectorial version of EVP (in short, VEVP) is considered and studied in $[5,22,23]$ and references therein. Aubin and Frankowska [4] presented the equilibrium version of EVP (in short, EEVP) in the setting of complete metric spaces. Such version of EVP is further studied in $[9,11,21]$ with applications to an equilibrium problem which is a unified model of several problems, namely, variational inequalities, complementarity problems, fixed point problem, optimization problem, Nash equilibrium problem, saddle point problem, and so forth; see, for example, $[24,25]$ and references therein. Al-Homidan et al. [9] established EEVP in the setting of quasimetric 
spaces with a $Q$-function which generalizes the notions of $\tau$-function [17] and a $w$-distance [8]. They proved some equivalences of EEVP with a fixed point theorem of Caristi-Kirk type for multivalued maps [12], Takahashi's minimization theorem [8], and some other related results. As applications, they derived the existence results for solutions of equilibrium problems and fixed point theorems for multivalued maps. They also extended the Nadler's fixed point theorem [26] for multivalued maps to a $Q$-function in the setting of complete quasimetric spaces. As a consequence, they proved the Banach contraction theorem for a $Q$ function in the setting of complete quasimetric spaces. Ansari [10] extended EEVP for vector valued functions in the setting of complete quasimetric spaces with a $W$-distance. By using this result, he derived the existence of solutions of a vector equilibrium problem [25], that is, equilibrium problem for vector valued functions. He established some equivalent results to EEVP for vector valued functions and also established Caristi-Kirk fixed point theorem for multivalued maps [12] in a more general setting.

In 1983, Dancs et al. [27] established a critical point theorem in the setting of complete metric spaces and proved EVP by using their result. Lin et al. $[18,19]$ used the critical point theorem of Dancs et al. [27] to establish EEVP for multivalued bifunctions. They also studied intersection theorems, variational inclusion problems, and some other related problems by using the critical point theorem of Dancs et al. [27]. Khanh and Quy [15] generalized the critical point theorem of Dancs et al. [27] in the setting of metric spaces with $\tau$-functions [17]. Khanh and Quy $[15,16]$ used their result to established EEVP for vector valued functions.

In this paper, we introduce the concepts of $\lambda$-function and $\lambda$-space which are much weaker than those of cone metric and cone metric space defined by Huang and X. Zhang [28], respectively. We establish some critical point theorems in the setting of $\lambda$-spaces and, in particular, in the setting of complete cone metric spaces. Our results generalize those results given in [15, 27]. As applications of our results, we characterize the completeness of cone metric spaces and give VEVP and EEVP for multivalued maps in the setting of cone metric spaces. Moreover, we improve and generalize many Ekeland type variational principles and critical point theorems.

\section{Preliminaries}

Let $E$ be a topological vector space with origin $\mathbf{0}$. For a given nontrivial, pointed, closed and convex cone $P \subset E$, we define on $E$ a partial ordering $\leq$ (resp., $<$ ) with respect to $P$ by $x \leq y$ (resp., $x<y$ ) if $y-x \in P$ (resp., $y-x \in P \backslash\{0\}$ ), and we denote by $x \ll y$ if $y-x \in \operatorname{int} P$ (interior of $P$ ). When $E$ is a normed space, the nontrivial, pointed, closed, and convex cone $P$ is said to be normal cone if there exists $K>0$ such that for all $x, y \in E$,

$$
\mathbf{0} \leq x \leq y \text { implies }\|x\| \leq K\|y\|
$$

The least positive number $K$ satisfying (2.1) is called the normal constant of $P$.

Throughout the paper, unless otherwise specified, we assume that $X$ is a nonempty set, $E$ is a real topological vector space with origin $\mathbf{0}$ ordered by a nontrivial, pointed, closed

and convex cone $P$ and $\lambda: X \times X \rightarrow E$ is a vector valued function. We denote by $2^{X}$ the family of all subsets of $X$. If $E$ is a normed space, for each $s \in E, r \in \mathbb{R}_{+}=[0, \infty)$, we use the following notations:

(i) $B(s, r)=\{y \in E:\|s-y\|<r\}$,

(ii) $B^{*}(s, r)=\{y \in E:\|s-y\|<r\} \backslash\{s\}$. 
Definition 2.1 (see [28]). Let $X$ be a nonempty set, and let $E$ be a topological vector space ordered by a nontrivial, pointed, and closed convex cone $P$. Let $d: X \times X \rightarrow E$ be a vector valued function such that the following conditions hold:

$$
\begin{aligned}
& \text { (d1) } 0 \leq d(x, y) \text { for all } x, y \in X \text { and } d(x, y)=0 \text { if and only if } x=y, \\
& (d 2) d(x, y)=d(y, x) \text { for all } x, y \in X, \\
& \text { (d3) } d(x, y) \leq d(x, z)+d(z, y) \text { for all } x, y, z \in X .
\end{aligned}
$$

Then $d$ is called a cone metric on $X$, and the set $X$ with a cone metric $d$ is called a cone metric space and it is denoted by $(X, d)$.

Definition 2.2. A vector valued function $\lambda: X \times X \rightarrow E$ is said to be a $\lambda$-function if for all $x, y \in X$,

$$
\begin{aligned}
& \text { ( 11) } \lambda(x, y) \geq \mathbf{0}, \\
& (\lambda 2) x \neq y \Rightarrow \lambda(x, y) \neq \mathbf{0} .
\end{aligned}
$$

A nonempty set $X$ with a $\lambda$-function is called a $\lambda$-space, and it is denoted by $(X, \lambda)$.

Clearly, every cone metric space is a $\lambda$-space, but the converse is not true; see below, Example 2.4.

Definition 2.3. (i) A sequence $\left\{x_{n}\right\}_{n \in \mathbb{N}}$ in a $\lambda$-space $(X, \lambda)$ is said to be the following:

(a) $\lambda$-Cauchy sequence (resp., quasi- $\lambda$-Cauchy sequence) if for every $c \in E$ with $c \gg \mathbf{0}$, there exists a positive integer $N$ such that $\lambda\left(x_{n}, x_{m}\right) \ll c$ (resp., $\left.\lambda\left(x_{n}, x_{m}\right)<c\right)$ for all $n, m \geq N$.

(b) $\lambda$-convergent (resp., quasi- $\lambda$-convergent) if there exists $x \in \mathrm{X}$ such that for every $c \in E$ with $c \gg 0$, there exists a positive integer $N$ such that $\lambda\left(x_{n}, x\right) \ll c$ (resp., $\left.\lambda\left(x_{n}, x\right)<c\right)$ for all $n \geq N$. In this case, we say that $\left\{x_{n}\right\} \lambda$-converges (resp., quasi$\lambda$-converges) to $x$ in $(X, \lambda)$, and we denote it by $x_{n} \stackrel{\jmath}{\rightarrow} x$ (resp., $x_{n} \stackrel{q-\lambda}{\longrightarrow} x$ ). The point $x \in X$ is called a $\lambda$-limit point (resp., quasi- $\lambda$-limit point) of the sequence $\left\{x_{n}\right\}$.

(ii) A $\lambda$-space $(X, \lambda)$ is said to be $\lambda$-complete (resp., quasi- $\lambda$-complete) if every $\lambda$-Cauchy sequence (resp., quasi- $\lambda$-Cauchy sequence) is a $\lambda$-convergent (resp., quasi- $\lambda$-convergent) sequence.

(iii) A subset $D$ of a $\lambda$-space $(X, \lambda)$ is said to be the following:

(a) $\lambda$-closed (resp., quasi- $\lambda$-closed) in $(X, \lambda)$ if for every $x \in X$ with a sequence $\left\{x_{n}\right\} \subset D$ such that $\left\{x_{n}\right\} \lambda$-converges (resp., quasi- $\lambda$-converges) to $x$ in $(X, \lambda)$, then $x \in D$; the $\lambda$-closure of a set $D$ in $(X, \lambda)$ is the intersection of all $\lambda$-closed sets containing $D$.

(b) $\lambda$-open (resp., quasi- $\lambda$-open) in $(X, \lambda)$ if $D^{c}=X \backslash D$ (the complement of $D$ in $X$ ) is $\lambda$-closed (resp., quasi- $\lambda$-closed).

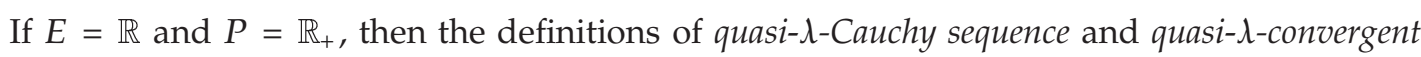
sequence are equal to the ones of $\lambda$-Cauchy sequence and $\lambda$-convergent sequence, respectively.

Example 2.4. Let $X=E=\mathbb{R}$ and $P=\mathbb{R}_{+}$. Define $\lambda: X \times X \rightarrow E$ by

$$
\lambda(x, y)= \begin{cases}|x-y| & \text { if } x, y \in \mathbb{Q} \text { (rational numbers) } \\ 1 & \text { otherwise. }\end{cases}
$$


Then, $\lambda$ is a $\lambda$-function and $(X, \lambda)$ is a $\lambda$-space. But $(X, \lambda)$ is not a cone metric space. Furthermore, the subset $\mathbb{Q}$ is $\lambda$-closed in $(X, \lambda)$.

Proof. If $x \in X$ with a net $\left\{x_{n}\right\}_{n \in \mathbb{N}} \subset \mathbb{Q}$ such that $x_{n} \stackrel{\jmath}{\rightarrow} x$, then $x \in \mathbb{Q}$. Indeed, suppose that $x \notin \mathbb{Q}$, then $\lambda\left(x_{n}, x\right)=1$ for all $n \in \mathbb{N}$. This contradicts the fact that $x_{n} \stackrel{\jmath}{\rightarrow} x$.

Lemma 2.5. If $x_{n} \stackrel{\curlywedge}{\rightarrow} x$, then for every subsequence $\left\{x_{n_{k}}\right\}$ of $\left\{x_{n}\right\}$, one has $x_{n_{k}} \stackrel{\curlywedge}{\rightarrow} x$. Similar results

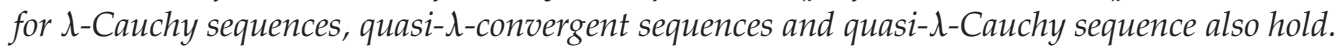

Proof. By definition, for any $c \gg 0$, there exists a positive integer $N$ such that $\lambda\left(x_{n}, x\right) \ll c$ for all $n \geq N$. For every subsequence $\left\{x_{n_{k}}\right\}$ of $\left\{x_{n}\right\}$, we have $\lambda\left(x_{n}, x\right) \ll c$ for all $n_{k} \geq N$ and hence $\left\{x_{n_{\mathrm{k}}}\right\}$ also $\lambda$-converges to $x$.

Remark 2.6. (a) If $(X, d)$ is a cone metric space, and if we replace $\lambda$ by $d$ in Definition 2.3 (i) and (ii), we obtain the definitions of a $d$-Cauchy sequence and $d$-convergent sequence in a cone metric space and the definition of a $d$-complete cone metric space in [28], respectively. If there is no danger of confusion, then we will not use the letter $d$ before these definitions.

(b) As it is proved in [28], every convergent sequence is a Cauchy sequence in a cone metric space. But this assertion is not true for a $\lambda$-convergent sequence in $(X, \lambda)$. For example, let $X=E=\mathbb{R}$ and $P=\mathbb{R}_{+}$. Define $\lambda: X \times X \rightarrow E$ by

$$
\lambda(x, y)= \begin{cases}|x-y| & \text { if } x=0 \text { or } y=0 \\ 1 & \text { otherwise }\end{cases}
$$

Then, $\{1 / n\}_{n \in \mathbb{N}}$ is a $\lambda$-convergent sequence with $\lambda$-limit 0 , but it is not a $\lambda$-Cauchy sequence.

(c) With the help of $\lambda$-open (resp., quasi- $\lambda$-open) sets and $\lambda$-closed (resp., quasi- $\lambda$ closed) sets, we can easily endow $X$ with topology which would be weaker than the topology generated by quasimetric spaces and cone metric spaces.

Proof. (i) It is obvious that the empty set $\emptyset$ and $X$ are $\lambda$-closed sets.

(ii) Let $A$ and $B$ be $\lambda$-closed sets, and let $\left\{x_{n}\right\} \subset A \cup B$ be a sequence such that $x_{n} \stackrel{\jmath}{\rightarrow} x$ for some $x \in X$ (Note that this $x$ may not be unique). For each such $x$ with $x_{n} \stackrel{\curlywedge}{\rightarrow} x$, without loss of generality, we can extract a subsequence $\left\{x_{n_{k}}\right\} \subset A$ of $\left\{x_{n}\right\}$. Since $\left\{x_{n_{k}}\right\} \lambda$-converges to $x$ in $(X, \lambda)$ and $A$ is $\lambda$-closed, then $x \in A \subset A \cup B$, and therefore the set $A \cup B$ is $\lambda$-closed.

(iii) Let $\left\{A_{i}\right\}_{i \in I}$ be any family of $\lambda$-closed sets, and let $\left\{y_{n}\right\} \subset \bigcap_{i \in I} A_{i}$ be any sequence which $\lambda$-converges to $y$ in $(X, \lambda)$. Then, for each $i \in I,\left\{y_{n}\right\} \subset A_{i}$ and $y_{n} \stackrel{\jmath}{\rightarrow} y \in A_{i}$ since $A_{i}$ is $\lambda$-closed. Therefore, $y \in \bigcap_{i \in I} A_{i}$, and hence $\bigcap_{i \in I} A_{i}$ is a $\lambda$-closed set. Therefore, $\lambda$-space $(X, \lambda)$ is a topological space with the topology consists of all $\lambda$-open sets.

The proof for the case of quasi- $\lambda$-closed sets or quasi- $\lambda$-open sets lies on the same lines of the above proof.

Definition 2.7. Let $A$ be a nonempty subset of a $\lambda$-space $(X, \lambda)$, and let $\left\{A_{n}\right\}$ be a sequence of nonempty subsets in $(X, \lambda)$. We adopt the following notations.

(i) $\delta(A)<c$ for some $c \in E$ with $c \geq 0$ if $\lambda(x, y)<c$ for all $x, y \in A$.

(ii) $\rho(A)=\sup \{\|\lambda(x, y)\|: x, y \in A\}$ if $E$ is a normed vector space with an ordered cone $P$. 
Fixed Point Theory and Applications

(iii) $\delta\left(A_{n}\right) \stackrel{\jmath}{\rightarrow} \mathbf{0}$ of the first type if for every $c \in E$ with $c>\mathbf{0}$, there exists a positive integer $N$ such that $\delta\left(A_{n}\right)<c$ for all $n \geq N$.

(iv) $\delta\left(A_{n}\right) \stackrel{\jmath}{\rightarrow} \mathbf{0}$ of the second type if for every $c \in E$ with $c \gg \mathbf{0}$, there exists a positive integer $N$ such that $\delta\left(A_{n}\right)<c$ for all $n \geq N$.

(v) $\delta\left(A_{n}\right) \stackrel{\jmath}{\rightarrow} \mathbf{0}$ of the first type w.r.t. $\left\{y_{n}\right\} \subseteq X$ if for every $c \in E$ with $c>\mathbf{0}$, there exists a positive integer $N$ such that for each $n \geq N$, we have $\lambda\left(y_{n}, u\right)<c$ for all $u \in A_{n}$.

(vi) $\delta\left(A_{n}\right) \stackrel{l}{\rightarrow} \mathbf{0}$ of the second type w.r.t. $\left\{y_{n}\right\} \subseteq X$ if for every $c \in E$ with $c \gg \mathbf{0}$, there exists a positive integer $N$ such that for each $n \geq N$, we have $\lambda\left(y_{n}, u\right)<c$ for all $u \in A_{n}$.

Now, we recall the definitions of $\tau$-functions and weak $\tau$-functions.

Definition 2.8 (see [17]). Let $(X, d)$ be a metric space. A function $p: X \times X \rightarrow \mathbb{R}_{+}$is said to be a $\tau$-function if the following conditions are satisfied.

$(\tau 1)$ For all $x, y, z \in X, p(x, z) \leq p(x, y)+p(y, z)$.

$(\tau 2) p(x, \cdot)$ is $\mathbb{R}_{+}$-lower semicontinuous, for each $x \in X$.

( $\tau 3)$ For any sequences $\left\{x_{n}\right\}$ and $\left\{y_{n}\right\}$ in $X$ with $\limsup _{n \rightarrow \infty}\left\{p\left(x_{n}, x_{m}\right): m>n\right\}=0$ and $\lim _{n \rightarrow \infty} p\left(x_{n}, y_{n}\right)=0$, one has $\lim _{n \rightarrow \infty} d\left(x_{n}, y_{n}\right)=0$.

$(\tau 4)$ For $x, y, z \in X, p(x, y)=0$, and $p(x, z)=0$ imply $y=z$.

Definition 2.9. Let $(X, d)$ be a quasimetric space (i.e., symmetricity is not required). A function $p: X \times X \rightarrow \mathbb{R}_{+}$is said to be a weak $\tau$-function if the conditions $(\tau 1),(\tau 3)$, and $(\tau 4)$ hold.

Remark 2.10. The definition of weak $\tau$-functions on a metric space is given in [15].

Definition 2.11 (see [27]). Let $F: X \rightarrow 2^{X}$ be a multivalued map. A point $x \in X$ is said to be a critical point of $F$ if and only if $F(x)=\{x\}$.

\section{Critical Point Theorems}

We present the following critical point theorem in the setting of $\lambda$-spaces.

Theorem 3.1. Let $(X, \lambda)$ be a quasi- $\lambda$-complete space, and let $F: X \rightarrow 2^{X}$ be a multivalued map with nonempty quasi- $\lambda$-closed values. Assume that

(i) for all $x, y \in X, y \in F(x)$ implies $F(y) \subseteq F(x)$,

(ii) for every sequence $\left\{x_{n}\right\}$ with $x_{n+1} \in F\left(x_{n}\right)$, one has $\delta\left(F\left(x_{n}\right)\right) \stackrel{\lambda}{\rightarrow} \mathbf{0}$ of the first type.

Then, for each $\hat{x} \in X$, there exists $x^{*} \in F(\widehat{x})$ such that $F\left(x^{*}\right)=\left\{x^{*}\right\}$ and $\lambda\left(x^{*}, x^{*}\right)=0$.

Proof. For any fixed $\hat{x} \in X$, let $x_{1}=\widehat{x}$ and take $x_{n+1} \in F\left(x_{n}\right)$ for all $n \in \mathbb{N}$. By conditions (i) and (ii), we have $F\left(x_{n+1}\right) \subseteq F\left(x_{n}\right)$ for all $n \in \mathbb{N}$ and $\delta\left(F\left(x_{n}\right)\right) \stackrel{\jmath}{\rightarrow} \mathbf{0}$ of the first type. So, for any $c \in E$ with $c>0$, there exists a positive integer $N$ such that

$$
\lambda\left(y_{n}, z_{n}\right)<c \quad \forall y_{n}, z_{n} \in F\left(x_{n}\right) \text { whenever } n \geq N \text {. }
$$


Therefore, $\left\{x_{n}\right\}$ is a quasi- $\lambda$-Cauchy sequence in $(X, \lambda)$. Since $(X, \lambda)$ is a quasi- $\lambda$-complete, $\left\{x_{n}\right\}$ quasi- $\lambda$-converges to some point $x$ in $(X, \lambda)$ (Note that the above quasi- $\lambda$-limit point $x$ may not be unique). For each quasi- $\lambda$-limit point $x^{*}$ of $\left\{x_{n}\right\}, x^{*} \in F\left(x_{n}\right)$ for all $n \in \mathbb{N}$ because $F\left(x_{n}\right)$ is quasi- $\lambda$-closed and $F\left(x_{n+1}\right) \subseteq F\left(x_{n}\right)$ for all $n \in \mathbb{N}$. Since $\delta\left(F\left(x_{n}\right)\right) \stackrel{\jmath}{\rightarrow} \mathbf{0}$ of the first type, we have

$$
\bigcap_{n \in \mathbb{N}} F\left(x_{n}\right)=\left\{x^{*}\right\}
$$

Indeed, if there exists $y \in \bigcap_{n \in \mathbb{N}} F\left(x_{n}\right)$ with $y \neq x^{*}$, let $c^{\prime}=\lambda\left(x^{*}, y\right)>0$. Since $\delta\left(F\left(x_{n}\right)\right) \stackrel{\jmath}{\rightarrow}$ 0 of the first type, there exists a positive integer $N^{\prime}$ such that $\delta\left(F\left(x_{n}\right)\right)<c^{\prime} / 2$ for all $n \geq N^{\prime}$. Then, $c^{\prime}=\lambda\left(x^{*}, y\right)<c^{\prime} / 2$. This leads to a contradiction. Therefore, $\emptyset \neq F\left(x^{*}\right) \subseteq \bigcap_{n \in \mathbb{N}} F\left(x_{n}\right)=$ $\left\{x^{*}\right\}$, and hence $F\left(x^{*}\right)=\left\{x^{*}\right\}$. Therefore, for each quasi-l-limit point $x^{*}$ of $\left\{x_{n}\right\}$, we have $F\left(x^{*}\right)=\bigcap_{n \in \mathbb{N}} F\left(x_{n}\right)=\left\{x^{*}\right\}$ and hence the net $\left\{x_{n}\right\}$ has a unique quasi- $\lambda$-limit point, say $\bar{x}$ with $F(\bar{x})=\{\bar{x}\}$. Further, since $\delta\left(F\left(x_{n}\right)\right) \stackrel{\curlywedge}{\rightarrow} \mathbf{0}$ of the first type, $\lambda(\bar{x}, \bar{x})=\mathbf{0}$.

Example 3.2. Let $X=E=\mathbb{R}$ and $P=\mathbb{R}_{+}$. Define $\lambda: X \times X \rightarrow E$ by

$$
\lambda(x, y)=\max \{|x|,|y|\}
$$

Then $(X, \lambda)$ is a $\lambda$-space but it is neither a cone metric space nor a quasimetric space. If we define a multivalued map $F$ on $X$ by

$$
F(x)= \begin{cases}\left(-\frac{|x|}{2}, \frac{|x|}{2}\right) & \text { if } x \neq 0 \\ \{0\} & \text { if } x=0\end{cases}
$$

Then, all the conditions of Theorem 3.1 are satisfied, and hence there exists a critical point of $F$ in $(X, \lambda)$, but neither [27, Theorem 3.1] nor [15, Lemma 3.4] is applicable in this example. In fact, $F(0)=\{0\}$ and $\lambda(0,0)=0$.

Remark 3.3. When $E=\mathbb{R}, P=\mathbb{R}_{+}$and $(X, \lambda)$ is a metric space, Theorem 3.1 reduces to Theorem 3.1 in [27]. say that

For a transitive relation $\mathfrak{R}$ (i.e., $x \Re y$ and $y \mathfrak{R} z$ imply $x \mathfrak{R} z$ ) in a topological space $Y$, we

(i) $\mathfrak{R}$ is lower closed if for any $\mathfrak{R}$-monotone (i.e., $\ldots \mathfrak{R} x_{n} \mathfrak{R} \ldots \mathfrak{R} x_{2} \mathfrak{R} x_{1}$ ) convergent sequence $x_{n} \rightarrow \bar{x}$ one has $\bar{x} \Re x_{n}$ for all $n \in \mathbb{N}$,

(ii) a subset $A \subseteq Y$ is $\mathfrak{R}$-complete if any Cauchy sequence in $A$ (if the definition of Cauchy sequence is given) which is $\Re$-monotone converges to a point of $A$.

Remark 3.4. If the relation $\Re$ on $(X, \lambda)$ in Theorem 3.1 is given by

$$
x \Re y \Longleftrightarrow x \in F(y) \quad \forall x, y \in X
$$


then the assumptions " $F$ has quasi- $\lambda$-closed values" and "quasi- $\lambda$-completeness of $(X, \lambda)$ " in Theorem 3.1 (in fact, in all the results of this paper) can be replaced by the assumptions " $R$ is lower closed" and " $F(x)$ is $\Re$-complete for all $x \in X$ ", respectively.

Proof. Construct a net $\left\{x_{\alpha}\right\}$ in the same manner as in Theorem 3.1. From conditions (i) and (ii) of Theorem 3.1, we have $x_{n+1} \Re x_{n}$ for all $n \in \mathbb{N}$ and $\delta\left(F\left(x_{n}\right)\right) \stackrel{\jmath}{\rightarrow} \mathbf{0}$ of the first type. So, for any $c \in E$ with $c>0$, there exists a positive integer $N$ such that

$$
\lambda\left(y_{n}, z_{n}\right)<c \quad \forall y_{n}, z_{n} \in F\left(x_{n}\right) \text { whenever } n \geq N
$$

Therefore, $\left\{x_{n}\right\}$ is a $\mathfrak{R}$-monotone quasi- $\lambda$-Cauchy sequence in $(X, \lambda)$. Since $(X, \lambda)$ is $\mathfrak{R}$ complete, there exists $\bar{x} \in X$ such that $\bar{x} \mathfrak{R} x_{n}$ for all $n \in \mathbb{N}$ (note that the above limit $\bar{x}$ may not be unique). Following the same argument as in the proof of Theorem 3.1, we obtain the conclusion.

Remark 3.5. In Theorem 3.1, if $(X, \lambda)$ is $\lambda$-complete (not necessarily quasi- $\lambda$-complete), $F$ has $\lambda$-closed values (not necessarily quasi- $\lambda$-closed values), and if we assume further that the vector space $E$ is a normed space, then the condition (ii) of Theorem 3.1 can be replaced by the following condition.

(ii) $^{\prime}$ For every sequence $\left\{x_{n}\right\}$ with $x_{n+1} \in F\left(x_{n}\right)$, we have $\lim _{n \rightarrow \infty} \rho\left(F\left(x_{n}\right)\right)=0$.

Proof. Let $\lambda^{\prime}: X \times X \rightarrow \mathbb{R}_{+}$be defined as

$$
\lambda^{\prime}(x, y)=\|\lambda(x, y)\| \quad \forall x, y \in X
$$

Then, a $\lambda^{\prime}$-Cauchy sequence in $\left(X, \lambda^{\prime}\right)$ is a $\lambda$-Cauchy sequence in $(X, \lambda)$.

For it, let $\left\{x_{n}\right\}$ be a $\lambda^{\prime}$-Cauchy sequence in $\left(X, \lambda^{\prime}\right)$. Since $c \gg 0$ means that $c \in \operatorname{int} P$, for each fixed $c \in E$ with $c \gg 0$, there exists $\varepsilon>0$ such that $B(c, \varepsilon) \subset \operatorname{int} P$. Since $\lim _{n \rightarrow \infty} \rho\left(F\left(x_{n}\right)\right)=0$, there exists a positive integer $N$ such that $\|\lambda(x, y)\|<\varepsilon$ for all $x, y \in F\left(x_{n}\right)$ whenever $n \geq N$. Then, $c-\lambda(x, y) \in \operatorname{int} P$ for all $x, y \in F\left(x_{n}\right)$ whenever $n \geq N$, and hence $\lambda(x, y) \ll c$ for all $x, y \in F\left(x_{n}\right)$ whenever $n \geq N$. Therefore, sequence $\left\{x_{n}\right\}$ is a $\lambda$-Cauchy sequence in $(X, \lambda)$.

Since $(X, \lambda)$ is $\lambda$-complete, a $\lambda^{\prime}$-Cauchy sequence in $\left(X, \lambda^{\prime}\right)$ is $\lambda$-convergent in $(X, \lambda)$.

For any fixed $\widehat{x} \in X$, by the same argument as in the proof of Theorem 3.1, there exists $x^{*} \in X$ such that $x^{*} \in F\left(x_{n}\right)$ for all $n \in \mathbb{N}$ with $x_{1}=\hat{x}$. Since $\lim _{n \rightarrow \infty} \rho\left(F\left(x_{n}\right)\right)=0$, we have

$$
\bigcap_{n \in \mathbb{N}} F\left(x_{n}\right)=\left\{x^{*}\right\}
$$

Indeed, if there exists $y \in \bigcap_{n \in \mathbb{N}} F\left(x_{n}\right)$ with $y \neq x^{*}$, let $c^{\prime}=\lambda\left(x^{*}, y\right)>0$, then

$$
\left\|c^{\prime}\right\|=\left\|\lambda\left(x^{*}, y\right)\right\| \leq \rho\left(F\left(x_{n}\right)\right) \quad \forall n \in \mathbb{N} .
$$

Since $\lim _{n \rightarrow \infty} \rho\left(F\left(x_{n}\right)\right)=0,0<\left\|c^{\prime}\right\| \leq 0$. This leads to a contradiction. Therefore, $\emptyset \neq F\left(x^{*}\right) \subseteq$ $\bigcap_{n \in \mathbb{N}} F\left(x_{n}\right)=\left\{x^{*}\right\}$ for all $\lambda$-limit points $x^{*}$ of $\left\{x_{n}\right\}$. Then, following the same argument as Theorem 3.1 to complete the proof. 
Lemma 3.6. Let $(X, \lambda)$ be a $\lambda$-space, and let $E$ be a normed vector space with an ordering cone $P$. Let $\left\{A_{n}\right\}$ be a sequence of subsets of $X$ such that $A_{n+1} \subseteq A_{n}$ for all $n \in \mathbb{N}$. Then, $\lim _{n \rightarrow \infty} \rho\left(A_{n}\right)=0$ implies $\delta\left(A_{n}\right) \stackrel{\lambda}{\rightarrow} \boldsymbol{0}$ of the second type in $(X, \lambda)$. Moreover, the converse holds if $P$ is a normal cone.

Proof. Let $\lim _{n \rightarrow \infty} \rho\left(A_{n}\right)=0$. For any fixed $c \in E$ with $c \gg 0$, there exists $\varepsilon>0$ such that $B(c, \varepsilon) \subset$ int $P$. Since $\lim _{n \rightarrow \infty} \rho\left(A_{n}\right)=0$, there exists a positive $N$ such that

$$
\|\lambda(x, y)\|<\varepsilon \quad \forall x, y \in A_{n} \text { whenever } n \geq N .
$$

Then,

$$
c-\lambda(x, y) \in \operatorname{int} P \quad \forall x, y \in A_{n} \text { whenever } n \geq N,
$$

and hence

$$
\lambda(x, y) \ll c \quad \forall x, y \in A_{n} \text { whenever } n \geq N
$$

Therefore, $\delta\left(A_{n}\right) \stackrel{\curlywedge}{\rightarrow} \mathbf{0}$ of the second type in $(X, \lambda)$.

Conversely, assume that $E$ is ordered by a normal cone $P$ and $\delta\left(A_{n}\right) \stackrel{\jmath}{\rightarrow} \mathbf{0}$ of the second type in $(X, \lambda)$. For any fixed $c^{\prime} \in E$ with $c^{\prime} \gg 0$, there exists a positive $N^{\prime}$ such that

$$
\lambda(x, y)<c^{\prime} \quad \forall x, y \in A_{n} \text { whenever } n \geq N^{\prime} .
$$

Then,

$$
\|\lambda(x, y)\| \leq K\left\|c^{\prime}\right\| \quad \forall x, y \in A_{n} \text { whenever } n \geq N^{\prime}
$$

where $K$ is the normal constant of $P$. Since $c^{\prime}$ was arbitrary, the proof is completed.

Lemma 3.7. Let $E$ be a normed vector space ordered by a normal cone $P$, then the following statements are equivalent.

(i) $\left\{x_{n}\right\}$ is a $\lambda$-Cauchy sequence in $(X, \lambda)$.

(ii) For every $\varepsilon>0$, there exists a positive $N$ such that $\left\|\lambda\left(x_{i}, x_{j}\right)\right\|<\varepsilon$ for all $i, j \geq N$.

Proof. It follows by taking $A_{n}=\left\{x_{k}\right\}_{k=n}^{\infty}$ in Lemma 3.6.

Remark 3.8. Note that the topological space $(X, d)$ in [28] is assumed to be a cone metric space. Therefore, Lemma 3.7 generalizes [28, Lemma 4] Example 3.9; see below. Further, as a consequence of Remark 3.4 and Lemma 3.6, if $E$ is assumed to be a normed vector space with a normal ordering cone $P,(X, \lambda)$ is $\lambda$-complete (not necessarily quasi- $\lambda$-complete) and the values of mapping $F$ is $\lambda$-closed (not necessarily quasi- $\lambda$-closed). Then, the condition (ii) of Theorem 3.1 can be replaced by the following.

(ii)" For every sequence $\left\{x_{n}\right\}$ with $x_{n+1} \in F\left(x_{n}\right)$, we have $\delta\left(F\left(x_{n}\right)\right) \stackrel{\curlywedge}{\rightarrow} \mathbf{0}$ of the second type. 
Example 3.9. In Example 3.2, $(X, \lambda)$ is a $\lambda$-space but not a cone metric space. By Lemma 3.7, $\{1 / n\}_{n \in \mathbb{N}}$ is a $\lambda$-Cauchy sequence but $[28$, Lemma 4$]$ is not applicable.

Now, we establish a critical point theorem in the setting of cone metric spaces.

Theorem 3.10. Let $(X, d)$ be a complete cone metric space, E a normed vector space, and $F: X \rightarrow 2^{X}$ a multivalued map with nonempty closed values. Assume that

(i) for all $x, y \in X, y \in F(x)$ implies $F(y) \subseteq F(x)$,

(ii) for every sequence $\left\{x_{n}\right\}$ with $x_{n+1} \in F\left(x_{n}\right)$, one has $\lim _{n \rightarrow \infty}\left\|d\left(x_{n}, x_{n+1}\right)\right\|=0$.

Then, for each $\hat{x} \in X$, there exists $x^{*} \in F(\widehat{x})$ such that $F\left(x^{*}\right)=\left\{x^{*}\right\}$.

Proof. Without loss of generality, we may assume that for each $x \in X, F(x)$ is bounded; that is, $\rho(F(x))$ exists. For any given $\varepsilon>0$ and any fixed element $\widehat{x} \in X$, let $x_{1}=\widehat{x}$ and choose $x_{2} \in F\left(x_{1}\right)$ such that

$$
\left\|d\left(x_{1}, x_{2}\right)\right\|>\frac{\rho\left(F\left(x_{1}\right)\right)}{2}-\frac{\varepsilon}{2}
$$

Continuing in this way, we obtain a sequence $\left\{x_{n}\right\}_{n \in \mathbb{N}}$ such that $x_{n+1} \in F\left(x_{n}\right)$ and

$$
\left\|d\left(x_{n}, x_{n+1}\right)\right\|>\frac{\rho\left(F\left(x_{n}\right)\right)}{2}-\frac{\varepsilon}{2^{n}}, \quad \forall n \in \mathbb{N}
$$

Since $\lim _{n \rightarrow \infty}\left\|d\left(x_{n}, x_{n+1}\right)\right\|=0$ and $\varepsilon$ is arbitrary positive number, we have

$$
\lim _{n \rightarrow \infty} \rho\left(F\left(x_{n}\right)\right)=0
$$

By Lemma 3.7, sequence $\left\{x_{n}\right\}_{n \in \mathbb{N}}$ is a Cauchy sequence in $(X, d)$. Since $(X, d)$ is complete, $\left\{x_{n}\right\}$ converges to some $x^{*} \in X$. By hypothesis, $F\left(x_{n}\right)$ is closed and $F\left(x_{n+1}\right) \subseteq F\left(x_{n}\right)$ for all $n \in \mathbb{N}$. Then, $x^{*} \in F\left(x_{n}\right)$ for all $n \in \mathbb{N}$. Since $\lim _{n \rightarrow \infty} \rho\left(F\left(x_{n}\right)\right)=0$, we have

$$
\bigcap_{n \in \mathbb{N}} F\left(x_{n}\right)=\lim _{n \rightarrow \infty} F\left(x_{n}\right)=\left\{x^{*}\right\}
$$

Indeed, if there exists $y \in \bigcap_{n \in \mathbb{N}} F\left(x_{n}\right)$ with $y \neq x^{*}$. Then,

$$
\begin{gathered}
0<\left\|d\left(x^{*}, y\right)\right\| \leq \rho\left(F\left(x_{n}\right)\right), \quad \forall n \in \mathbb{N}, \\
\rho\left(F\left(x_{n}\right)\right) \longrightarrow 0 \quad \text { as } n \longrightarrow \infty .
\end{gathered}
$$

This leads to a contradiction. Therefore, $\emptyset \neq F\left(x^{*}\right) \subseteq \bigcap_{n \in \mathbb{N}} F\left(x_{n}\right)=\left\{x^{*}\right\}$, and hence $F\left(x^{*}\right)=$ $\left\{x^{*}\right\}$. 
Remark 3.11. We observe that if $E$ is a normed space and $\left\{A_{n}\right\}_{n \in \mathbb{N}}$ is a sequence of subsets in a cone metric space $(X, d)$ with $A_{n+1} \subseteq A_{n}$ for all $n \in \mathbb{N}$, then the following statements are equivalent:

(a) $\lim _{n \rightarrow \infty} \rho\left(A_{n}\right)=0$,

(b) for any sequence $\left\{a_{n}\right\}$ with $a_{n} \in A_{n}, \lim _{n \rightarrow \infty}\left\|d\left(a_{n}, a_{n+1}\right)\right\|=0$.

The following result characterizes the $\lambda$-completeness of a $\lambda$-space.

Theorem 3.12. Let $(X, \lambda)$ be a $\lambda$-space, $E$ a normed space ordered by a normal cone $P$, and $F: X \rightarrow$ $2^{X}$ a multivalued map with nonempty $\lambda$-closed values. Assume that

(i) for all $x, y \in X, y \in F(x)$ implies $F(y) \subseteq F(x)$,

(ii) for every sequence $\left\{x_{n}\right\}$ with $x_{n+1} \in F\left(x_{n}\right)$, one has $\delta\left(F\left(x_{n}\right)\right) \stackrel{\jmath}{\rightarrow} \mathbf{0}$ of the second type in $(X, \lambda)$,

(iii) for all $x, y, z \in X, \lambda(x, z) \leq \lambda(x, y)+\lambda(y, z)$.

Then, the multivalued map $F$ has a critical point in $X$ if and only if $(X, \lambda)$ is $\lambda$-complete.

Proof. The sufficiency follows from Remark 3.5 and Lemma 3.6 (condition (iii) is not used).

We now show the necessity. Let $\left\{x_{n}\right\}_{n \in \mathbb{N}}$ be a $\lambda$-Cauchy sequence in $(X, \lambda)$. If $x \in$ ${\overline{\left\{x_{n}\right\}}}_{n \in \mathbb{N}}$ (the $\lambda$-closure of $\left\{x_{n}\right\}_{n \in \mathbb{N}}$ in $(X, \lambda)$ ), then by condition (iii), either $x=x_{n}$ for some $n \in \mathbb{N}$ or $x$ is a $\lambda$-limit point of $\left\{x_{n}\right\}_{n \in \mathbb{N}}$. Let $A_{n}=\overline{\left\{x_{k}\right\}_{k=n}^{\infty}}$ (the $\lambda$-closure of $\left\{x_{k}\right\}_{k=n}^{\infty}$ in $(X, \lambda)$ ). For each $x \in{\overline{\left\{x_{n}\right\}}}_{n \in \mathbb{N}}$, we consider the following two cases:

(i) $x \in\left\{x_{n}\right\}_{n \in \mathbb{N}}$, then $x=x_{k} \in A_{k} \neq \emptyset$ for some $k \in \mathbb{N}$,

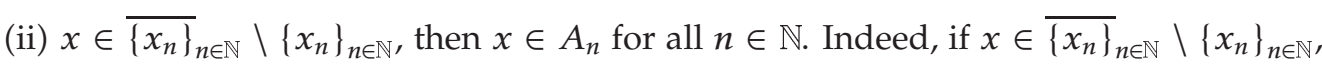
then $x$ is a $\lambda$-limit point of $\left\{x_{n}\right\}_{n \in \mathbb{N}}$, and hence $x \in A_{n}$ for all $n \in \mathbb{N}$. In this case, $x \in \bigcap_{n \in \mathbb{N}} A_{n} \neq \emptyset$.

Define a multivalued mapping $F: \overline{\left\{x_{n}\right\}} \rightarrow 2^{\overline{\left\{x_{n}\right\}}}$ by

$$
F(x)= \begin{cases}A_{n} & \text { if } x=x_{n} \text { for some } n \in \mathbb{N}, \\ \bigcap_{n \in \mathbb{N}} A_{n} & \text { otherwise. }\end{cases}
$$

Then, $x \in F(x) \neq \emptyset$ for all $x \in{\overline{\left\{x_{n}\right\}}}_{n \in \mathbb{N}}$.

Since $\left\{x_{n}\right\}_{n \in \mathbb{N}}$ is a $\lambda$-Cauchy sequence in $(X, \lambda)$, then for each $c \gg 0$, there exists $N \in \mathbb{N}$ such that $\lambda\left(x_{n}, x_{m}\right) \ll c / 2$ for all $m, n \geq N$. Then, by condition (iii), for each $n>N$ and $x, y \in A_{n}$, we have

$$
\lambda(x, y) \leq \lambda\left(x, x_{n}\right)+\lambda\left(x_{n}, y\right) \ll \frac{c}{2}+\frac{c}{2}=c .
$$

Then, $\delta\left(A_{n}\right) \stackrel{\lambda}{\rightarrow} \mathbf{0}$ of the second type in $(X, \lambda)$. For each $\left\{u_{n}\right\}_{n \in \mathbb{N}}$ in $\overline{\left\{x_{n}\right\}}$ with $u_{n+1} \in F\left(u_{n}\right)$ for all $n \in \mathbb{N}$, we consider the following two cases. 
Case 1. $\left\{u_{n}\right\}_{n \in \mathbb{N}}$ is a subsequence of $\left\{x_{n}\right\}_{n \in \mathbb{N}}$.

Case 2. there exists $n \in \mathbb{N}$ such that $u_{n} \in{\overline{\left\{x_{n}\right\}_{n \in \mathbb{N}}}} \backslash\left\{x_{n}\right\}_{n \in \mathbb{N}}$.

In Case $1, \delta\left(F\left(u_{n}\right)\right)=\delta\left(F\left(x_{n_{k}}\right)\right) \stackrel{\jmath}{\rightarrow} \mathbf{0}$ of the second type for some subsequence $\left\{x_{n_{k}}\right\}$ of $\left\{x_{n}\right\}_{n \in \mathbb{N}}$. In Case 2, $F\left(u_{n}\right)=\bigcap_{k \in \mathbb{N}} A_{k}$ for some $n \in \mathbb{N}$. Since $\delta\left(A_{n}\right) \stackrel{\jmath}{\rightarrow} \mathbf{0}$ of the second type in $(X, \lambda), \delta\left(F\left(u_{n}\right)\right) \stackrel{\jmath}{\rightarrow} \mathbf{0}$ of the second type. Then, the condition (ii) is satisfied.

For each $x, y \in{\overline{\left\{x_{n}\right\}}}_{n \in \mathbb{N}}$ with $y \in F(x)$, we consider the following two cases.

Case 1. $x=x_{n}$ for some $n \in \mathbb{N}$, then $y=x_{m}$ for some $m \geq n$ or $y$ is a $\lambda$-limit point of $\left\{x_{n}\right\}_{n \in \mathbb{N}}$. Then $F(x)=A_{n}$ and $F(y)=A_{m}$ for some $m \geq n$ or $F(y)=\bigcap_{n \in \mathbb{N}} A_{n}$.

Case 2. $x$ is a $\lambda$-limit point of $\left\{x_{n}\right\}_{n \in \mathbb{N}}$, then $y$ is also a $\lambda$-limit point of $\left\{x_{n}\right\}_{n \in \mathbb{N}}$. Then, $F(x)=$ $F(y)=\bigcap_{n \in \mathbb{N}} A_{n}$.

In either cases, we have that $F(y) \subseteq F(x)$ and condition (i) is satisfied. By assumption, there exists a critical point of $F$, say $x^{*} \in X$. Then, $x^{*} \in A_{n}$ for all $n \in \mathbb{N}$. Indeed, if $x^{*}=x_{n}$ for some $n \in \mathbb{N}$, then $\left\{x_{n}\right\}=\left\{x^{*}\right\}=F\left(x^{*}\right)=F\left(x_{n}\right)=A_{n}$. Then, $x_{m}=x^{*}=x_{n}$ for all $m \geq n$ and hence $x^{*} \in A_{n}=\bigcap_{k \in \mathbb{N}} A_{k}$. If $x^{*} \neq x_{n}$ for all $n \in \mathbb{N}$, then $x^{*} \in \bigcap_{n \in \mathbb{N}} A_{n}$. In either cases, we have that $x^{*} \in \bigcap_{n \in \mathbb{N}} A_{n}$. Since $P$ is normal and $\delta\left(A_{n}\right) \stackrel{\jmath}{\rightarrow} \mathbf{0}$ of the second type in $(X, \lambda)$, for each $\varepsilon>0$, there exists $N \in \mathbb{N}$ such that $\left\|\lambda\left(x_{n}, x^{*}\right)\right\|<\varepsilon$ for all $n \geq \mathbb{N}$ by Lemma 3.6. Since for every fixed $c \gg 0$, there exists $\delta>0$ such that $B(c, \delta) \subseteq \operatorname{int} P$ and so $c-\lambda\left(x_{n}, x^{*}\right) \in \operatorname{int} P$ as $n$ large enough. Hence, $\lambda\left(x_{n}, x^{*}\right) \ll c$ as $n$ large enough. Therefore, the sequence $\left\{x_{n}\right\} \lambda$-converges to $x^{*}$ in $(X, \lambda)$.

Corollary 3.13. Let $(X, d)$ be a cone metric space, $E$ a normed vector space ordered by a normal cone $P$, and $F: X \rightarrow 2^{X}$ be a multivalued map with nonempty closed values in $(X, d)$. Suppose that

(i) for all $x, y \in X, y \in F(x)$ implies $F(y) \subseteq F(x)$,

(ii) $\delta\left(A_{n}\right) \stackrel{d}{\rightarrow} \mathbf{0}$ of the second type in $(X, d)$.

Then, $F$ has a critical point in $X$ if and only if $(X, d)$ is d-complete. [15].

We next establish another critical point theorem which generalizes the main result in

Theorem 3.14. Let $(X, \lambda)$ be a quasi- $\lambda$-complete space, and let $F: X \rightarrow 2^{X}$ be a multivalued map with nonempty quasi- $\lambda$-closed values. Suppose that

(i) for all $x, y \in X, y \in F(x)$ implies $F(y) \subseteq F(x)$,

(ii) $\left\{x_{n}\right\}_{n \in \mathbb{N}}$ is a sequence in $(X, \lambda)$ with $x_{n+1} \in F\left(x_{n}\right)$, and there exists $\left\{y_{n}\right\}_{n \in \mathbb{N}}$ with $y_{n} \in$ $F\left(x_{n}\right)$ such that $\delta\left(F\left(x_{n}\right)\right) \stackrel{\jmath}{\rightarrow} \mathbf{0}$ of the first type w.r.t. $\left\{y_{n}\right\}_{n \in \mathbb{N}}$ in $(X, \lambda)$,

(iii) every quasi-ᄉ-convergent net in $(X, \lambda)$ has a unique limit.

Then there exists $x^{*} \in F\left(x_{1}\right)$ such that $F\left(x^{*}\right)=\left\{x^{*}\right\}$.

Proof. Let $\left\{x_{n}\right\}$ and $\left\{y_{n}\right\}$ be the sequences given by condition (ii). If $m, n \in \mathbb{N}, m>n$, by (i), we have $y_{m} \in F\left(x_{m}\right) \subseteq F\left(x_{n}\right)$. Since $\delta\left(F\left(x_{n}\right)\right) \stackrel{\curlywedge}{\rightarrow} \mathbf{0}$ of the first type w.r.t. $\left\{y_{n}\right\}_{n \in \mathbb{N}}$ in $(X, \lambda)$, for every $c \in E$ with $c>0$, there exists a positive integer $N$ such that $\lambda\left(y_{n}, y_{m}\right)<c$ whenever $m, n \geq N$. 
Therefore, $\left\{y_{n}\right\}$ is a quasi- $\lambda$-Cauchy sequence in $(X, \lambda)$. Since $(X, \lambda)$ is quasi- $\lambda$-complete, $\left\{y_{n}\right\}$ quasi- $\lambda$-converges to some $x^{*} \in X$. Since $F\left(x_{n}\right)$ is quasi- $\lambda$-closed and $F\left(x_{n+1}\right) \subseteq F\left(x_{n}\right)$ for all $n \in \mathbb{N}$, we have $x^{*} \in F\left(x_{n}\right)$ for all $n \in \mathbb{N}$. Since $\delta\left(F\left(x_{n}\right)\right) \stackrel{\jmath}{\rightarrow} \mathbf{0}$ of the first type w.r.t. $\left\{y_{n}\right\}$ in $(X, \lambda)$, we obtain $F\left(x^{*}\right)=\left\{x^{*}\right\}$. Indeed, for each $y \in F\left(x^{*}\right) \subseteq \bigcap_{n \in \mathbb{N}} F\left(x_{n}\right)$, since $\delta\left(F\left(x_{n}\right)\right) \stackrel{\curlywedge}{\rightarrow} 0$ of the first type w.r.t. $\left\{y_{n}\right\}$ in $(X, \lambda)$, both $x^{*}$ and $y$ are quasi- $\lambda$-limit points of $\left\{y_{n}\right\}_{n \in \mathbb{N}}$. Since the limit is assumed to be unique, $x^{*}=y$ and $F\left(x^{*}\right)=\left\{x^{*}\right\}$.

Remark 3.15. The uniqueness assumption of the limit in Theorem 3.14 holds if for each $x \in X$, one of the following conditions is satisfied:

(A) $\lim _{n \rightarrow \infty} \lambda\left(x_{n}, x\right)=0$ implies $\lim _{n \rightarrow \infty} \lambda\left(x, x_{n}\right)=\mathbf{0}$ and for each $y \in X$, either $\lambda(x, y) \leq$ $\lambda(x, z)+\lambda(z, y)$ or $\lambda(y, x) \leq \lambda(y, z)+\lambda(z, x)$ for all $z \in X$,

(B) $\lambda(x, x)=0$ and for each $y \in X, \lambda(x, y) \leq \lambda(z, x)+\lambda(z, y)$ for all $z \in X$.

Indeed, it is obvious that condition (B) implies the symmetricity of $\lambda$ and thus implies condition (A). If condition (A) holds and both $x$ and $y$ are quasi- $\lambda$-limit of sequence $\left\{x_{n}\right\}_{n \in \mathbb{N}}$. We have $\lambda\left(x_{n}, x\right) \stackrel{q-\lambda}{\longrightarrow} 0$ and $\lambda\left(x_{n}, y\right) \stackrel{q-\lambda}{\longrightarrow} \mathbf{0}$. Further, either

$$
\lambda(x, y) \leq \lambda\left(x, x_{n}\right)+\lambda\left(x_{n}, y\right) \stackrel{q-\lambda}{\longrightarrow} 0 \text { as } n \longrightarrow \infty,
$$

or

$$
\lambda(y, x) \leq \lambda\left(y, x_{n}\right)+\lambda\left(x_{n}, x\right) \stackrel{q-\lambda}{\longrightarrow} 0 \quad \text { as } n \longrightarrow \infty .
$$

In either cases, we have $x=y$.

Now, we provide an example which shows that limit in a quasimetric spaces can be not unique.

Example 3.16. Let $X=[0,1]$ and define $d: X \times X \rightarrow \mathbb{R}_{+}$by

$$
d(x, y)= \begin{cases}y-x & \text { if } y \geq x \\ 1+y-x & \text { if } y<x \text { but }(x, y) \neq(1,0) \\ 1 & \text { if }(x, y)=(1,0)\end{cases}
$$

Obviously, for each $x, y \in X$, we have $d(x, y) \geq 0$ and $d(x, y)=0$ if and only if $x=y$. We show that $d(x, y) \leq d(x, z)+d(z, y)$ for all $x, y, z \in X$. Consider the following three cases:
(a) $x<y$,
(b) $x>y$ but $(x, y) \neq(1,0)$,
(c) $(x, y)=(1,0)$. 
For Case (a)

If $x<z<y$, then

$$
d(x, y)=y-x=(z-x)+(y-z)=d(x, z)+d(z, y),
$$

if $z<x<y$, then

$$
d(x, y)=y-x<y-z=d(z, y) \leq d(x, z)+d(z, y),
$$

if $x<y<z$, then

$$
d(x, y)=y-x<z-x=d(x, z) \leq d(x, z)+d(z, y) .
$$

For Case (b)

If $x>z>y$, then

$$
d(x, y)=1+y-x<1+z-x=d(x, z) \leq d(x, z)+d(z, y),
$$

if $z>x>y$, then

$$
d(x, y)=1+y-x=(z-x)+(1+y-z)=d(x, z)+d(z, y)
$$

if $x>y>z$, then

$$
d(x, y)=1+y-x=(1-x+z)+(y-z)=d(x, z)+d(z, y) .
$$

\section{For Case (c)}

For any $z \in(0,1)$,

$$
d(x, y)=1=z+(1-z)=d(x, z)+d(z, y) .
$$

Since $d(0,1 / 3)=1 / 3 \neq 2 / 3=d(1 / 3,0),(X, d)$ is a quasimetric space but not a metric space.

Further, for every sequence $\left\{x_{n}\right\} \subseteq[0,1)$ increasing to 1 , we have

$$
d\left(x_{n}, 1\right)=1-x_{n} \longrightarrow 0, \quad d\left(x_{n}, 0\right)=1-x_{n} \longrightarrow 0 .
$$

Then, both 1 and 0 are limits of the convergent sequence $\left\{x_{n}\right\}$ in $(X, d)$, but $1 \neq 0$ in $(X, d)$ for $d(1,0)=d(0,1)=1 \neq 0$. Therefore, limit in a quasimetric space $(X, d)$ is not always unique. 
The following example shows that in the setting of quasimetric spaces condition (A) in Remark 3.11 is a reasonable assumption.

Example 3.17. Let $X=\mathbb{R}$ and define $d: X \times X \rightarrow \mathbb{R}_{+}$by

$$
d(x, y)= \begin{cases}y-x & \text { if } x \leq y \\ -2(y-x) & \text { if } x>y\end{cases}
$$

Then, $(X, d)$ is a quasimetric space, but not a metric space. Further, it is easy to verity that condition (A) in Remark 3.15 holds.

Remark 3.18. In Theorems 3.1 and 3.14, the assumptions " $(X, \lambda)$ is quasi- $\lambda$-complete" and " $F$ has nonempty quasi- $\lambda$-closed values" is imposed to guarantee quasi- $\lambda$-limit $x^{*}$ of a sequence $\left\{x_{n}\right\} \subset X$ such that $x^{*} \in \bigcap_{n \in \mathbb{N}} F\left(x_{n}\right)$. Therefore, these assumptions can be replaced by the following condition: there exists $\bar{x} \in X$ such that $\bar{x} \in F(x)$ for all $x \in X$.

Indeed, for any fixed $\hat{x} \in X$, take a sequence $\left\{x_{n}\right\}_{n=1}^{\infty}$ with $x_{1}=\hat{x}$ and $x_{n+1} \in F\left(x_{n}\right)$ for all $n \in \mathbb{N}$, we have $\bar{x} \in \bigcap_{n \in \mathbb{N}} F\left(x_{n}\right)$. By the same argument as in the proof of Theorem 3.1 (also, Theorem 3.14), we see that $F(\bar{x})=\{\bar{x}\}$.

As a corollary of Theorem 3.14 and Remark 3.15, we have the following result, which generalizes one of the main tools in $[15,16]$.

Corollary 3.19. Let $(X, d)$ be a quasimetric space having a unique limit of a convergent sequence, $p$ a weak $\tau$-function on $X$, and $F: X \rightarrow 2^{X}$ a multivalued map. Assume that $\left\{x_{n}\right\} \subseteq X$ converges to $\bar{x} \in X$ so that the following conditions are satisfied:

(i) $x_{n+1} \in F\left(x_{n}\right)$ and $F\left(x_{n+1}\right) \subseteq F\left(x_{n}\right)$ for all $n \in \mathbb{N}$,

(ii) $\limsup _{n \rightarrow \infty}\left\{p\left(x_{n}, u\right): u \in F\left(x_{n}\right)\right\}=0$,

(iii) $\bar{x} \in F\left(x_{n}\right)$ for all $n \in \mathbb{N}$.

then, $\bigcap_{n \in \mathbb{N}} F\left(x_{n}\right)=\{\bar{x}\}$.

If, in addition,

(iv) $F(\bar{x}) \neq \emptyset$ and $F(\bar{x}) \subseteq F\left(x_{n}\right)$ for all $n \in \mathbb{N}$,

then $F(\bar{x})=\{\bar{x}\}$.

Proof. Let $E=\mathbb{R}$ with $P=\mathbb{R}_{+}$and define a multivalued mapping $G: F\left(x_{1}\right) \rightarrow 2^{F\left(x_{1}\right)}$ by

$$
G(x)= \begin{cases}F\left(x_{n}\right), & \text { if } x \in F\left(x_{n}\right) x \notin F\left(x_{m}\right) \forall m>n, \\ \bigcap_{n \in \mathbb{N}} F\left(x_{n}\right), & \text { if } x \in \bigcap_{n \in \mathbb{N}} F\left(x_{n}\right) .\end{cases}
$$

As an application of Theorem 3.14 and Remark 3.15, it suffices to show that the conditions (i) and (ii) implies $\delta\left(F\left(x_{n}\right)\right) \rightarrow \mathbf{0}$ of the first type w.r.t. $\left\{x_{n}\right\}$ in $(X, d)$. First, we show that for each sequence, $\left\{u_{n}\right\} \subseteq X$ such that $u_{n} \in F\left(x_{n}\right)$, we have $\lim _{n \rightarrow \infty} d\left(x_{n}, u_{n}\right)=0$. 
Indeed, since $\lim \sup _{n \rightarrow \infty}\left\{p\left(x_{n}, u\right): u \in F\left(x_{n}\right)\right\}=0$, we have

$$
\begin{gathered}
\lim _{n \rightarrow \infty} p\left(x_{n}, u_{n}\right) \leq \limsup _{n \rightarrow \infty}\left\{p\left(x_{n}, u\right): u \in F\left(x_{n}\right)\right\}=0, \\
\limsup _{n \rightarrow \infty}\left\{p\left(x_{n}, x_{m}\right): m>n\right\} \leq \limsup _{n \rightarrow \infty}\left\{p\left(x_{n}, u\right): u \in F\left(x_{n}\right)\right\}=0 .
\end{gathered}
$$

By $(\tau 3), \lim _{n \rightarrow \infty} d\left(x_{n}, u_{n}\right)=0$.

We will show that $\lim _{n \rightarrow \infty} \sup _{u \in F\left(x_{n}\right)} d\left(x_{n}, u\right)=0$. Suppose to the contrary that there exists $\varepsilon>0$ and sequence $\left\{u_{n_{k}}\right\}$ with $u_{n_{k}} \in F\left(x_{n_{k}}\right)$ such that for each $n_{k}$, we have $d\left(x_{n_{k}}, u_{n_{k}}\right) \geq$ $\varepsilon$. This contradicts the fact that $\lim _{k \rightarrow \infty} d\left(x_{n_{k}}, u_{n_{k}}\right)=0$. Since $G\left(x_{n}\right) \subseteq F\left(x_{n}\right)$ for all $n \in \mathbb{N}$ and $\lim _{n \rightarrow \infty} \sup _{u \in F\left(x_{n}\right)} d\left(x_{n}, u\right)=0$, then $\delta\left(G\left(x_{n}\right)\right) \rightarrow 0$ (is of the first type) w.r.t. $\left\{x_{n}\right\}_{n \in \mathbb{N}}$ in $(X, d)$ and $x_{n} \in G\left(x_{n}\right)$ for all $n \in \mathbb{N}$. By the definition of mapping $G$, for all $x, y \in X$ with $y \in G(x)$, we have $G(y) \subseteq G(x)$. Indeed, $G(x)=F\left(x_{n}\right)$ and $G(y)=F\left(x_{k}\right)$ for some $n, k \in \mathbb{N}$. Since $y \in G(x)=F\left(x_{n}\right)$, then by the definition of mapping $G$, we have that $k \geq n$. Then, $G(y)=$ $F\left(x_{k}\right) \subseteq F\left(x_{n}\right)=G(x)$. Then, by Theorem 3.14 and Remark 3.15., there exists $x^{*} \in X$ such that $G\left(x^{*}\right)=\left\{x^{*}\right\}$. Since $\bar{x} \in \bigcap_{n \in \mathbb{N}} F\left(x_{n}\right) \subseteq G\left(x^{*}\right)$, then $\bar{x}=x^{*}$. Therefore $\bigcap_{n \in \mathbb{N}} F\left(x_{n}\right)=\{\bar{x}\}$.

Remark 3.20. In Corollary 3.19., if $(X, d)$ is a metric space, then Corollary 3.19. reduces to Lemma 3.4 in [15]. In the proof of Corollary 3.19, we have shown that the condition (ii) of Corollary 3.19 implies condition (ii) of Theorem 3.14. Khanh and Quy [16] showed that if a sequence $\left\{x_{n}\right\}$ is asymptotic by $p$, then $\left\{x_{n}\right\}$ satisfies condition (ii) of Corollary 3.19.

\section{Ekeland Type Variational Principles}

Definition 4.1. Let $(X, d)$ be a metric space. An extended real-valued function $f: X \rightarrow$ $(-\infty,+\infty]$ is said to be lower semicontinuous from above (in short, lsca) at $x_{0} \in X$ if for any sequence $\left\{x_{n}\right\}$ in $X$ with $x_{n} \rightarrow x_{0}$ and $f\left(x_{1}\right) \geq f\left(x_{2}\right) \geq \cdots \geq f\left(x_{n}\right) \geq \cdots$ imply that $f\left(x_{0}\right) \leq \lim _{n \rightarrow \infty} f\left(x_{n}\right)$. The function $f$ is said to be lsca on $X$ if $f$ is lsca at every point of $X$.

Definition 4.2. Let $E$ be a normed vector space ordered by a cone $P$. Then, we have the following.

(i) $E$ is called well-normed with respect to $P$ if there exists $S \in \mathbb{R}_{+}$such that

$$
\sum_{k=1}^{n}\left\|v_{k}\right\| \leq S\left\|\sum_{k=1}^{n} v_{k}\right\| \quad \forall n \in \mathbb{N} \text {, where } v_{k} \in P \forall k \in \mathbb{N} \text {. }
$$

The least positive number $S$ satisfying inequality (4.1) is called well-normed constant of $P$.

(ii) $E$ satisfies condition $(L)$ if for all $n \in \mathbb{N}$,

$$
\sum_{k=1}^{n} v_{k} \leq v \quad \text { for some } v \in E \text {, where } v_{k} \in P \forall k \in \mathbb{N} \text {, }
$$

then $\lim _{n \rightarrow \infty}\left\|v_{n}\right\|=0$. 
Example 4.3. For each $n \in \mathbb{N}$, let $\mathbb{R}^{n}$ be ordered by the cone $P=\mathbb{R}_{+}^{n}$. Then, $\mathbb{R}^{n}$ is not only well-normed with well norm constant $S=\sqrt{n}$ but also satisfies condition $(L)$.

Definition 4.4 (see [20]). Let $X$ be a topological space, $E$ be a topological vector space ordered by a cone $P$, and $F: X \rightarrow 2^{E}$ a multivalued map and $\operatorname{dom}(F)=\{x \in X: F(x) \neq \emptyset\}$. F is said to be

(i) upper P-continuous at $\bar{x} \in \operatorname{dom}(F)$ if for any neighborhood $V$ of the origin $\mathbf{0}$ of $E$, there exists a neighborhood $U$ of $\bar{x}$ such that $F(x) \subseteq F(\bar{x})+V+P$ for all $x \in$ $U \cap \operatorname{dom}(F)$,

(ii) lower P-continuous at $\bar{x} \in \operatorname{dom}(F)$ if for any neighborhood $V$ of the origin 0 of $E$, there exists a neighborhood $U$ of $\bar{x}$ such that $F(\bar{x}) \subseteq F(x)+V-P$ for all $x \in$ $U \cap \operatorname{dom}(F)$.

Remark 4.5 (see [20]). Let $X$ be a topological space, and let $f: X \rightarrow \mathbb{R}$ be a function. It can be easily verified that the followings statements are equivalent:

(i) $f$ is lower semicontinuous at $\bar{x}$,

(ii) $f$ is upper $\mathbb{R}_{+}$-continuous at $\bar{x}$,

(iii) $f$ is lower $\mathbb{R}_{+}$-continuous at $\bar{x}$.

The following lemma plays an important role in this section. For the sake of completeness of the paper, we give a detailed proof of this lemma.

Lemma 4.6 (see [20]). Let X be a topological space, E a topological vector space ordered by a cone $P$ and $G$, and $H: X \rightarrow 2^{E}$ multivalued maps with nonempty values. If $G$ is a lower $(-P)$-continuous map and $H$ is an upper P-continuous map with compact values, then the set $S=\{x \in X: G(x) \subseteq$ $H(x)+P\}$ is a closed set.

Proof. Let $x_{0} \in T=S^{c}$ (the complement of $S$ ) and $z_{0} \in G\left(x_{0}\right)$ such that $z_{0} \notin H\left(x_{0}\right)+P$. Since $H\left(x_{0}\right)+P$ is closed, there exists a balanced neighborhood $V$ of $\mathbf{0} \in E$ such that

$$
\left(z_{0}+V\right) \cap\left(H\left(x_{0}\right)+V+P\right)=\emptyset .
$$

Since $P$ is a cone, it follows that

$$
\left(z_{0}+V-P\right) \cap\left(H\left(x_{0}\right)+V+P\right)=\emptyset .
$$

By the upper $P$-continuity of $H$, there exists a neighborhood $U$ of $x_{0}$ such that

$$
H(x) \subseteq H\left(x_{0}\right)+V+P \quad \forall x \in U,
$$

and hence

$$
H(x)+P \subseteq H\left(x_{0}\right)+V+P \quad \forall x \in U .
$$


By lower $(-P)$-continuity of $G$, we may assume that $U$ is such that

$$
G\left(x_{0}\right) \subseteq G(x)+V+P \quad \forall x \in U
$$

Since $z_{0} \in G\left(x_{0}\right)$, this shows that for all $x \in U$, we can find $z \in G(x)$ such that $z \in z_{0}-V-P$. Then, for each $x \in U$, we can find $z \in G(x)$ such that $z \notin H(x)+P$. Thus, $U$ is contained in $T$, and $S$ is closed.

We present a version of Ekeland type variational principle in the setting of complete cone metric spaces.

Theorem 4.7. Let $E$ be a well-normed vector space ordered by a normal cone $P$. Let $(X, d)$ be a complete cone metric space, and let $f: X \rightarrow E$ be a lower $(-P)$-continuous function, bounded from below by $l$. Then, for every $\varepsilon>0$ and for every $\widehat{x} \in X$, there exists $x^{*} \in X$ such that

(i) $f\left(x^{*}\right)+\varepsilon d\left(x^{*}, \widehat{x}\right) \leq f(\widehat{x})$,

(ii) $\varepsilon d\left(x^{*}, x\right) \not f\left(x^{*}\right)-f(x)$ for all $x \in X \backslash\left\{x^{*}\right\}$.

Proof. Without loss of generality, we may assume that $\varepsilon=1$. Let

$$
F(x)=\{y \in X: f(y)+\varepsilon d(x, y) \leq f(x)\} \quad \forall x \in X,
$$

and take

$$
x_{1}=\widehat{x}, \quad x_{n+1} \in F\left(x_{n}\right) \quad \forall n \in \mathbb{N} .
$$

It is easy to verify that for each fixed $x \in X, d(x, \cdot)$ is an upper $P$-continuous function on $X$ and thus $-d(x, \cdot)$ is upper $(-P)$-continuous. Since $f$ is a lower $(-P)$-continuous function, by Lemma 4.6, $F$ is a multivalued map with nonempty closed values. Since $E$ is well-normed, we have

$$
\begin{aligned}
\sum_{k=1}^{\infty}\left\|d\left(x_{k}, x_{k+1}\right)\right\| & =\lim _{n \rightarrow \infty} \sum_{k=1}^{n}\left\|d\left(x_{k}, x_{k+1}\right)\right\| \\
& \leq \lim _{n \rightarrow \infty} K \sum_{k=1}^{n}\left\|f\left(x_{k}\right)-f\left(x_{k+1}\right)\right\| \\
& \leq \lim _{n \rightarrow \infty} S K\left\|\sum_{k=1}^{n} f\left(x_{k}\right)-f\left(x_{k+1}\right)\right\| \\
& \leq S K^{2}\left\|f\left(x_{1}\right)-l\right\|<\infty .
\end{aligned}
$$

Then, $\left\|d\left(x_{i}, x_{j}\right)\right\| \rightarrow 0$ as $i, j \rightarrow \infty$. Therefore, the conclusion follows from Theorem 3.10.

Now, we establish the following Ekeland type variational principle for multivalued bifunctions in the setting of complete cone metric spaces. 
Theorem 4.8. Let $(X, d)$ be a complete cone metric space, E be a normed vector space with an ordering cone $P$ that satisfies condition $(L)$, and let $F: X \times X \rightarrow 2^{E}$ and $H: X \rightarrow 2^{X}$ be multivalued maps. For each $x \in X$, suppose that the following conditions hold:

(i) $H(x)$ is a closed subset of $X$ and $H(y) \subseteq H(x)$ for all $y \in H(x)$,

(ii) There exists $y \in H(x)$ such that $F(x, y)+d(x, y) \subseteq-P$,

(iii) Either $F(x, \cdot)$ is bounded from below on $H(x)$ or $\bigcap_{y \in H(x)} F(x, y) \neq \emptyset$,

(iv) $F(x, z) \subseteq F(x, y)+F(y, z)-P$ for all $y \in H(x)$ and $z \in H(y)$;

(v) the map $F(x, \cdot)$ is lower P-continuous on $H(x)$.

Then, for every $\varepsilon>0$ and for every $\widehat{x} \in X$, there exists $x^{*} \in H(\widehat{x})$ such that
(a) $x^{*} \in H\left(x^{*}\right)$,
(b) $F\left(\widehat{x}, x^{*}\right)+\varepsilon d\left(\widehat{x}, x^{*}\right) \subseteq-P$,
(c) $F\left(x^{*}, x\right)+\varepsilon d\left(x^{*}, x\right) \nsubseteq-P$ for all $x \in H\left(x^{*}\right) \backslash\left\{x^{*}\right\}$.

Proof. Without loss of generality, we may assume that $\varepsilon=1$. For all $x \in X$, let

$$
G(x)=\{y \in X: F(x, y)+d(x, y) \subseteq-P\}, \quad S(x)=G(x) \cap H(x) .
$$

It is easy to verify that for each fixed $x \in X, d(x, \cdot)$ is an upper $P$-continuous function on $X$, thus $-d(x, \cdot)$ is upper $(-P)$-continuous. By condition (v) and Lemma 4.6, $S(x)$ is a closed subset of $H(x)$ for all $x \in X$. Since $H(x)$ is a closed subset of $X$ for all $x \in X, S(x)$ is also a closed subset of $X$ for all $x \in X$. For every $y \in S(x)$,

$$
F(x, y)+d(x, y) \subseteq-P .
$$

For every $z \in S(y)$,

$$
F(y, z)+d(y, z) \subseteq-P
$$

Then,

$$
F(x, z)+d(x, z) \subseteq F(x, y)+F(y, z)+d(x, y)+d(y, z)-P \subseteq-P .
$$

Hence, $S(y) \subseteq S(x)$ if $y \in S(x)$.

For arbitrary $\hat{x} \in X$, take the sequence $\left\{x_{n}\right\}_{n \in \mathbb{N}}$ with $x_{n+1} \in S\left(x_{n}\right)$ for all $n \in \mathbb{N}$ and $x_{1}=\hat{x}$. We have

$$
F\left(x_{n}, x_{n+1}\right)+d\left(x_{n}, x_{n+1}\right) \subseteq-P \quad \forall n \in \mathbb{N},
$$

therefore,

$$
\sum_{k=1}^{n} F\left(x_{k}, x_{k+1}\right)+\sum_{k=1}^{n} d\left(x_{k}, x_{k+1}\right) \subseteq-P
$$


By condition (iv),

$$
F\left(x_{1}, x_{n+1}\right)+\sum_{k=1}^{n} d\left(x_{k}, x_{k+1}\right) \subseteq \sum_{k=1}^{n} F\left(x_{k}, x_{k+1}\right)+\sum_{k=1}^{n} d\left(x_{k}, x_{k+1}\right)-P \subseteq-P .
$$

Hence, by condition (iii), there exists $l \in E$ such that $\sum_{k=1}^{n} d\left(x_{k}, x_{k+1}\right) \in l-P$. By condition $(L)$, we have $\lim _{n \rightarrow \infty}\left\|d\left(x_{k}, x_{k+1}\right)\right\|=0$. By Theorem 3.10, there exists $x^{*} \in S(\widehat{x})$ such that $S\left(x^{*}\right)=\left\{x^{*}\right\}$.

If $H(x)=X$ for all $x \in X$, then we deduce the following corollary from Theorem 4.8.

Corollary 4.9. Let $(X, d)$ be a complete cone metric space, $E$ a normed vector space with an ordering cone $P$ that satisfies condition (L), and $F: X \times X \rightarrow 2^{E}$ be a multivalued map. For each $x \in X$, suppose that the following conditions hold:

(i) there exists $y \in X$ such that $F(x, y)+d(x, y) \subseteq-P$,

(ii) either $F(x, \cdot)$ is bounded from below on $X$ or $\bigcap_{y \in X} F(x, y) \neq \emptyset$,

(iii) $F(x, z) \subseteq F(x, y)+F(y, z)-P$ for all $y, z \in X$,

(iv) the map $F(x, \cdot)$ is lower P-continuous on $X$.

Then, for every $\varepsilon>0$ and for every $\widehat{x} \in X$, there exists $x^{*} \in X$ such that

(a) $F\left(\widehat{x}, x^{*}\right)+\varepsilon d\left(\widehat{x}, x^{*}\right) \subseteq-P$,

(b) $F\left(x^{*}, x\right)+\varepsilon d\left(x^{*}, x\right) \nsubseteq-P$ for all $x \in X \backslash\left\{x^{*}\right\}$.

As an application of Theorem 3.12, we improve Theorem 5.1 in [17].

Theorem 4.10. Let $(X, d)$ be a metric space, $f: X \rightarrow \mathbb{R}$ a lsca function, $\varphi:(-\infty, \infty] \rightarrow \mathbb{R}_{+} a$ nondecreasing function, and $p$ a $\tau$-function on $X$. Define a binary relation $\mathfrak{R}$ on $X$ by

$$
y \Re x \Longleftrightarrow p(x, y) \leq \varphi(f(x))(f(x)-f(y))
$$

Suppose that $H: X \rightarrow 2^{X}$ is a multivalued map with nonempty values, $X$ is $\Re$-complete and $f$ is bounded from below on $X$, and for each $x \in X$, there exists $y \in H(x)$ such that $p(x, y) \leq$ $\varphi(f(x))(f(x)-f(y))$. Then, for each $u \in X$, there exists $v \in X$ such that

(i) $p(u, v) \leq \varphi(f(u))(f(u)-f(v))$,

(ii) $p(v, y)>\varphi(f(v))(f(v)-f(y))$ for all $y \in X, y \neq v$,

(iii) $v \in H(v)$.

Proof. Let $F(x)=\{y \in X: y \Re x\}, x_{1}=u$ and choose $x_{n+1} \Re x_{n}$ for all $n \in \mathbb{N}$. Then, it is easy to verify that $\mathfrak{R}$ is a transitive relation and hence condition (i) of Theorem 3.12 is satisfied. Let $\lim _{n \rightarrow \infty} x_{n}=v$. Since $f$ is lsca, the transitive relation $\mathfrak{R}$ is lower closed. By the same arguments in the proof of Theorem 4.7, we see that $\left\{x_{n}\right\}$ is asymptotic by $p$. As an application of Theorem 3.12 and Remarks 3.3 and 3.18, we complete the proof.

Remark 4.11. In [17, Theorem 5.1], the function $p$ is assumed to be a strong $\tau$-function (i.e., $p(x, y)>p(x, x)$ for all $x, y \in X$ with $x \neq y)$ and $p(x, x)=0$ for all $x \in X$. But as an application 
(Theorem 4.10) of Theorem 3.12, the assumptions above are not necessary and the proof is much easier.

Remark 4.12. Theorems 4.7-4.10 are generalizations of EVP [2]. To the best of our knowledge, there is almost no EVP results in the setting of cone metric spaces.

\section{Acknowledgments}

In this research, the third author was partially supported by the SABIC/Fast Track Research Project no. SB080004 of King Fahd University of Petroleum and Minerals, Dhahran, Saudi Arabia. His part was done during his stay at KFUPM, Dhahran.

\section{References}

[1] I. Ekeland, "Sur les problèmes variationnels," Comptes Rendus de l'Académie des Sciences, vol. 275, pp. A1057-A1059, 1972.

[2] I. Ekeland, "On the variational principle," Journal of Mathematical Analysis and Applications, vol. 47, pp. 324-353, 1974.

[3] I. Ekeland, "Nonconvex minimization problems," Bulletin of the American Mathematical Society, vol. 1, no. 3, pp. 443-474, 1979.

[4] J.-P. Aubin and H. Frankowska, Set-Valued Analysis, vol. 2 of Systems $\mathcal{E}$ Control: Foundations $\mathcal{E}$ Applications, Birkhäuser, Boston, Mass, USA, 1990.

[5] G.-Y. Chen, X. Huang, and X. Yang, Vector OptimizationOptimization: Set-Valued and Variational Analysis, vol. 541 of Lecture Notes in Economics and Mathematical Systems, Springer, Berlin, Germany, 2005.

[6] F. Facchinei and J.-S. Pang, Finite Dimensional Variational Inequalities and Complementarity Problems, vol. 1, Springer, Berlin, Germany, 2003.

[7] A. Göpfert, H. Riahi, C. Tammer, and C. Zălinescu, Variational Methods in Partially Ordered Spaces, Springer, New York, NY, USA, 2003.

[8] Wataru Takahashi, Nonlinear Functional Analysis, Yokohama Publishers, Yokohama, Japan, 2000.

[9] S. Al-Homidan, Q. H. Ansari, and J.-C. Yao, "Some generalizations of Ekeland-type variational principle with applications to equilibrium problems and fixed point theory," Nonlinear Analysis. Theory, Methods E Applications, vol. 69, no. 1, pp. 126-139, 2008.

[10] Q. H. Ansari, "Vectorial form of Ekeland-type variational principle with applications to vector equilibrium problems and fixed point theory," Journal of Mathematical Analysis and Applications, vol. 334, no. 1, pp. 561-575, 2007.

[11] M. Bianchi, G. Kassay, and R. Pini, "Existence of equilibria via Ekeland's principle," Journal of Mathematical Analysis and Applications, vol. 305, no. 2, pp. 502-512, 2005.

[12] J. Caristi and W. A. Kirk, "Geometric fixed point theory and inwardness conditions," in The Geometry of Metric and Linear Spaces, vol. 490, pp. 74-83, Springer, Berlin, Germany, 1975.

[13] J.-X. Fang, "The variational principle and fixed point theorems in certain topological spaces," Journal of Mathematical Analysis and Applications, vol. 202, no. 2, pp. 398-412, 1996.

[14] A. H. Hamel, "Equivalents to Ekeland's variational principle in uniform spaces," Nonlinear Analysis. Theory, Methods E Applications, vol. 62, no. 5, pp. 913-924, 2005.

[15] P. Q. Khanh and D. N. Quy, “On generalized Ekeland's variational principle and equivalent formulations for set-valued mappings," Journal of Global Optimization. In press.

[16] P. Q. Khanh and D. N. Quy, "A generalized distance and enhanced Ekeland's variational principle for vector functions," Nonlinear Analysis. Theory, Methods E Applications, vol. 73, no. 7, pp. 2245-2259, 2010.

[17] L.-J. Lin and W.-S. Du, "Ekeland's variational principle, minimax theorems and existence of nonconvex equilibria in complete metric spaces," Journal of Mathematical Analysis and Applications, vol. 323, no. 1, pp. 360-370, 2006.

[18] L.-J. Lin and C.-S. Chuang, "Existence theorems for variational inclusion problems and the set-valued vector Ekeland variational principle in a complete metric space," Nonlinear Analysis. Theory, Methods $\mathcal{E}$ Applications, vol. 70, no. 7, pp. 2665-2672, 2009. 
[19] L.-J. Lin, C.-S. Chuang, and S.-Y. Wang, "From quasivariational inclusion problems to Stampacchia vector quasiequilibrium problems, Stampacchia set-valued vector Ekeland's variational principle and Caristi's fixed point theorem," Nonlinear Analysis. Theory, Methods E Applications, vol. 71, no. 1-2, pp. 179-185, 2009.

[20] L. J. Lin and P. H. Sach, "Systems of generalized quasivariational inclusion problems with weak convexity and weak continuity and variants of set-valued vector Ekeland's variational principle," in Fixed Point Theory and its Applications, L. J. Lin, A. Petrusel, and H. K. Xu, Eds., pp. 115-129, Yokohama Publisher, Yokohama, Japan, 2010.

[21] W. Oettli and M. Théra, "Equivalents of Ekeland's principle," Bulletin of the Australian Mathematical Society, vol. 48, no. 3, pp. 385-392, 1993.

[22] C. Tammer, "A generalization of Ekeland's variational principle," Optimization, vol. 25, no. 2-3, pp. 129-141, 1992.

[23] A. B. Németh, "A nonconvex vector minimization problem," Nonlinear Analysis. Theory, Methods $\mathcal{E}$ Applications, vol. 10, no. 7, pp. 669-678, 1986.

[24] E. Blum and W. Oettli, "From optimization and variational inequalities to equilibrium problems," The Mathematics Student, vol. 63, no. 1-4, pp. 123-145, 1994.

[25] F. Giannessi, Vector Variational Inequalities and Vector Equilibria, vol. 38 of Mathematical Theories, Kluwer Academic, Dodrecht, The Netherlands, 2000.

[26] S. B. Nadler, Jr., "Multi-valued contraction mappings," Pacific Journal of Mathematics, vol. 30, pp. 475488, 1969.

[27] S. Dancs, M. Hegedús, and P. Medvegyev, "A general ordering and fixed-point principle in complete metric space," Acta Scientiarum Mathematicarum, vol. 46, no. 1-4, pp. 381-388, 1983.

[28] L.-G. Huang and X. Zhang, "Cone metric spaces and fixed point theorems of contractive mappings," Journal of Mathematical Analysis and Applications, vol. 332, no. 2, pp. 1468-1476, 2007. 